\title{
Lead Compound
}

National Cancer Institute

\section{Source}

National Cancer Institute. Lead Compound. NCI Thesaurus. Code C44395.

An inorg anic or org anic substance that contains the metallic element lead either in its positive-2 or 4 valence state. Lead compounds have a wide variety of applications in industry. Exposure to lead compounds affects the lungs, central nervous system and kidneys. Lead compounds are possible mutagens and are reasonably anticipated to be human carcinogens. The substance may be associated with an increased risk of developing lung and stomach cancer. ( $\mathrm{NCl05)}$ 